\title{
A thermopile detector array with scaled TE elements for use in an integrated IR microspectrometer
}

\author{
H Wu, S Grabarnik, A Emadi, G de Graaf and R F Wolffenbuttel \\ Faculty EEMCS, Department ME/EI, Delft University of Technology, Mekelweg 4, \\ 2828 CD Delft, The Netherlands \\ E-mail: H.W.Wu@tudelft.nl
}

Received 12 December 2007, in final form 28 April 2008

Published 19 May 2008

Online at stacks.iop.org/JMM/18/064017

\begin{abstract}
The design and fabrication of a thermopile detector array for use in a fully integrated infrared optical spectrometer are described. IC-compatible MEMS technologies are used for fabrication of the spectrometer components, such as the slit, planar imaging diffraction grating and detector array. The IR micro-spectrometer was designed for operation in the 1.5-3 $\mu \mathrm{m}$ wavelength range with the size of the largest dimension about $8 \mathrm{~mm}$. The imaging properties of the diffraction grating result in non-uniform dispersion, which imposes special requirements on the dimensions of each single detector in the array. The result is an array of unequally sized elements. The design considers technological constraints, sensitivity and cross-talk between elements. Simulation results, final design, fabrication technique and fabricated devices are presented.
\end{abstract}

(Some figures in this article are in colour only in the electronic version)

\section{Introduction}

Microsystems for measuring an optical spectrum have huge potential in many applications, such as chemistry, medicine, space, agriculture and quality inspection in manufacturing industries [1]. Compact spectrometers for the visible range with a limited spectral resolution and based on a transmission grating have been demonstrated in the visible [2] and infrared [3] spectral range. Resolving power is limited to $R=\lambda / \Delta \lambda=$ 20 , due to the lack of integrated optical components such as collimating lenses. Co-integration of optical components in a CMOS compatible fashion is difficult to achieve. Improved performance without jeopardizing IC compatibility is feasible when using a planar imaging diffraction grating. Due to the imaging properties of such a grating it is possible to combine collimating, dispersion and focusing in a single optical element, thus simplifying the spectrometer design considerably.

Imaging gratings are relatively easy to implement in waveguide-based systems and compact microspectrometer are presented in the literature [4-8]. However, implementation of a planar diffraction grating in a classical type of spectrometer has also been demonstrated [9]. A typical characteristic of a planar imaging diffraction grating is the maximum resolution at a selected central wavelength and a reduced spectral resolution for wavelengths away from this central wavelength with a minimum at the edges of the optical band considered. This property dictates the design of an array of detectors onto which the image is projected, since the pitch between adjacent elements should be kept to the minimum at the centre of the array and may increase further away from the centre for exploiting the spectral resolution provided by the grating. As a consequence, larger area detectors should be used at the edges of the spectral band in order not to reduce detectivity. This property could conveniently be used in a design by matching the array of detector elements of a nonuniform pitch to that of an array of readout channels with a constant pitch in such a way that the total array length is the same as minimum.

This paper is about the design and fabrication of an integrated infrared (IR) microspectrometer based on an imaging diffraction grating and an appropriately designed infrared detector array for application in the 1.5-3 $\mu \mathrm{m}$ wavelength range. The detector array design should take 


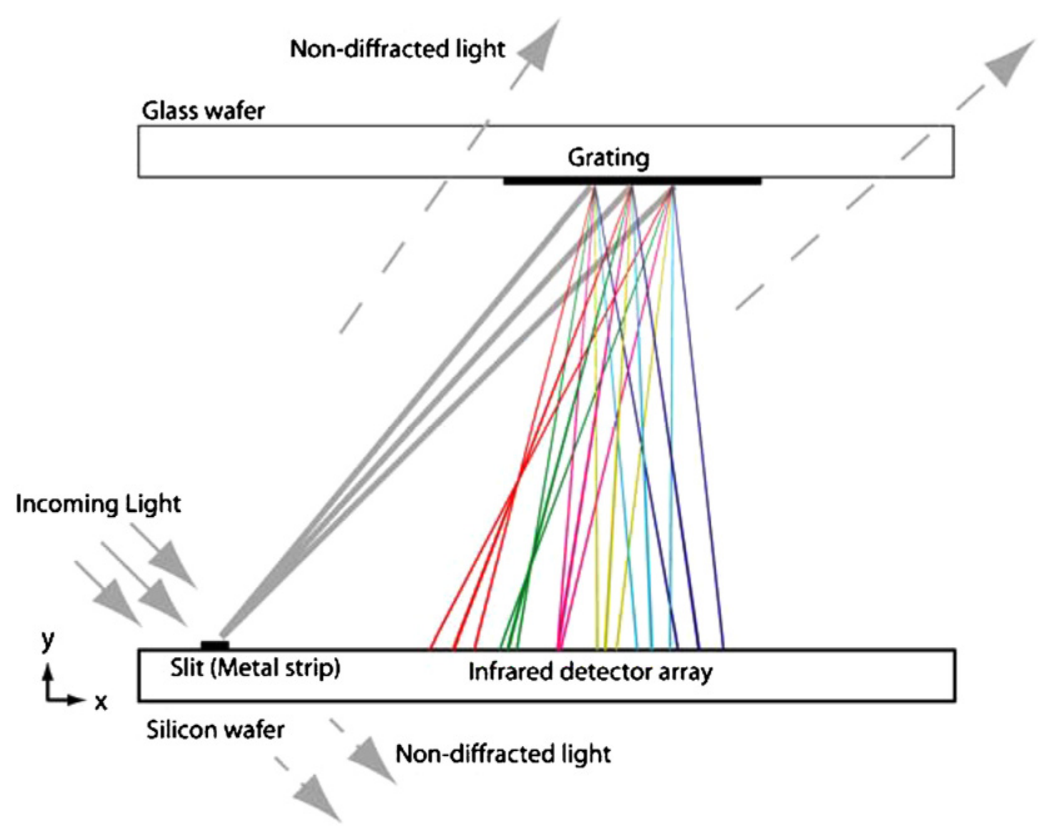

Figure 1. Structure of the planar diffraction imaging grating based IR microspectrometer.

technological limitations into account and should be adapted to the wavelength-dependent resolution of the spectral pattern produced by the diffraction grating. Based on these optical specifications and fabrication limitations, the geometry and materials of the detector array are determined and further optimized through an analytical model. The structures considered for the sensing element in the array are cantilever and bridge, which are to be realized on a membrane. A nitride membrane structure fabricated using MEMS technologies enables effective thermal isolation, which highly contributes to the sensitivity of the detector. The constraints imposed by the IC compatibility are included. An analytical model has been used to find the optimal parameters, such as the length of a thermopile, in a single detector. Other dimensions are dictated by the specific optical resolution. Once all the parameters that define the dimension of a single detector element are determined, finite element modelling (FEM) is used to calculate the expected performance with higher accuracy in the next step. The device is fabricated using a standard CMOS process, followed by $\mathrm{KOH}$ wet etching and subsequent reactive ion etching (RIE) to separate elements.

\section{Thermopile detector array design}

\subsection{The optical system}

The structure of the compact planar IR spectrometer is shown in figure 1. The spectrometer consists of two glass plates aligned parallel to each other. All spectrometer optical components, including the input slit and the diffraction grating, work in reflection. The incident light is reflected by a metal strip, which is thus acting as a slit, and is redirected towards the diffraction imaging grating fabricated on the upper glass plate. The diffracted light is finally projected on a chip that contains an array of thermo-electric elements.
The diffraction imaging grating is basically composed of a pattern of concentric circular grooves in a reflective metal with varying pitch. The grating can be designed to yield maximum spectral resolution at a specified design wavelength. As a consequence the spectral resolution reduces at wavelengths away from this design target, which in this design is at $2.25 \mu \mathrm{m}$. Perfect imaging is in principle achieved at this particular wavelength, as is confirmed by the centre beam incident on the detector array in the figure. The commercial optical design software ZEMAX [10] was used for the diffraction grating design and for calculation of the spectral resolution at different wavelengths. The result can be generally presented in the form of a spot diagram as shown in figure 2 . When the input slit of the spectrometer is illuminated by a monochromatic light source, the spot in this diagram provides an estimation of the full width of the image formed by the optical system. Since it would be pointless to have a detector pitch better than this resolution, the information in figure 2 determines the positiondependent pitch of the elements in the detector array.

\subsection{Theoretical fundamentals of the thermo-electric detector array}

The infrared thermopile detector used is based on the Seebeck effect. Incident light on the detectors heats up the absorption region and results in a temperature gradient, which is converted into voltage. The advantages as compared to resistive bolometers are: the intrinsic measurement of a temperature difference, the self-generating effect and the fact that selfheating due to readout is avoided. CMOS compatible Seebeck have already been investigated [11]. Material combinations with a high Seebeck coefficient should be selected to yield a maximum voltage at a given temperature gradient, however without increasing the thermal conduction. This performance 


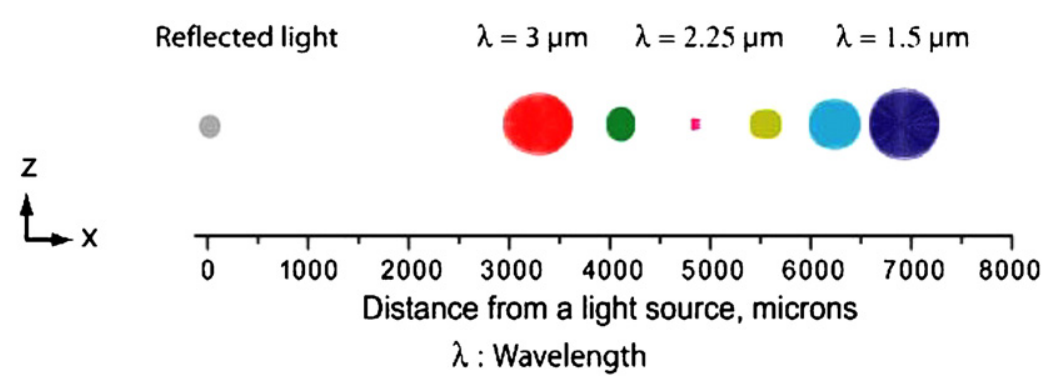

Figure 2. ZEMAX simulation result of the relative spot size.

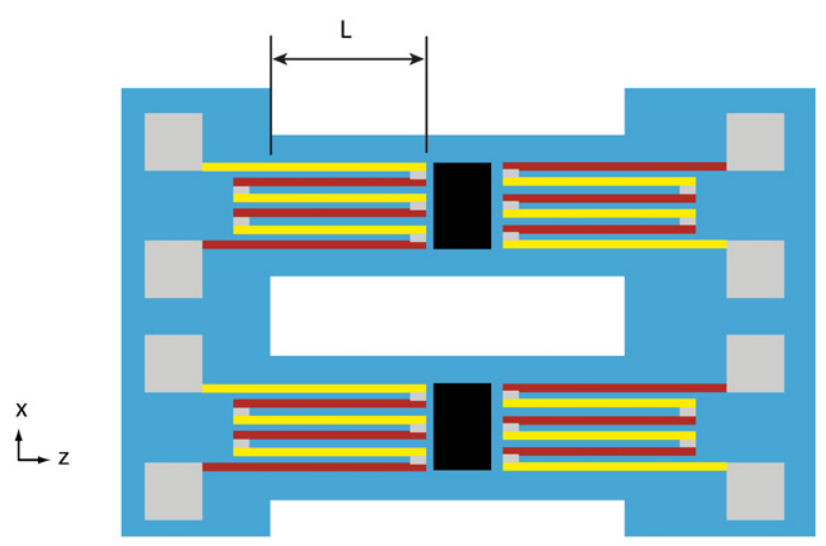

(a)

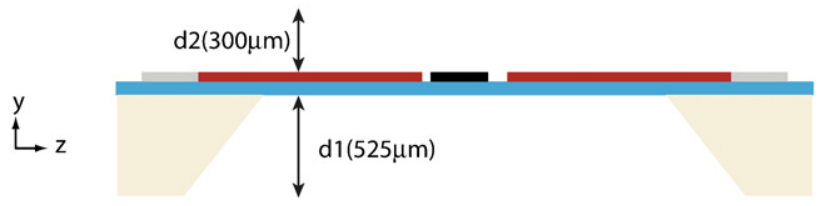

(b)

Figure 3. Thermopile bridge shape; the bridge is a SiN membrane made by bulk micromachining. Thermocouples are n-poly and p-poly.

is expressed in the material-dependent figure of merit, $Z$. Materials such as BiTe have higher Seebeck coefficient; however, they are not compatible with IC-processes and are more difficult to incorporate in a MEMS fabrication process [12]. Therefore, the temperature difference is highly depending on the geometries of the detector. IC-compatible MEMS technologies can be used to realize cantilever structures and bridge structures (a bridge is basically a symmetric double cantilever). These are the most suitable in this application and the bridge is shown in figure 3. Thermocouple pairs are formed on a bridge, which is cut out of a membrane by bulk micromachining, with an absorption area in the middle and thermocouples on both sides. In the structural sense this is a mechanical bridge, which is the starting point of any stress analysis. In the thermal sense this is a central heat source with two identical conduction paths. The analytical model allows, under some assumptions, the calculation of the thermopile performance and enables optimization of the geometry of a single sensing detector.

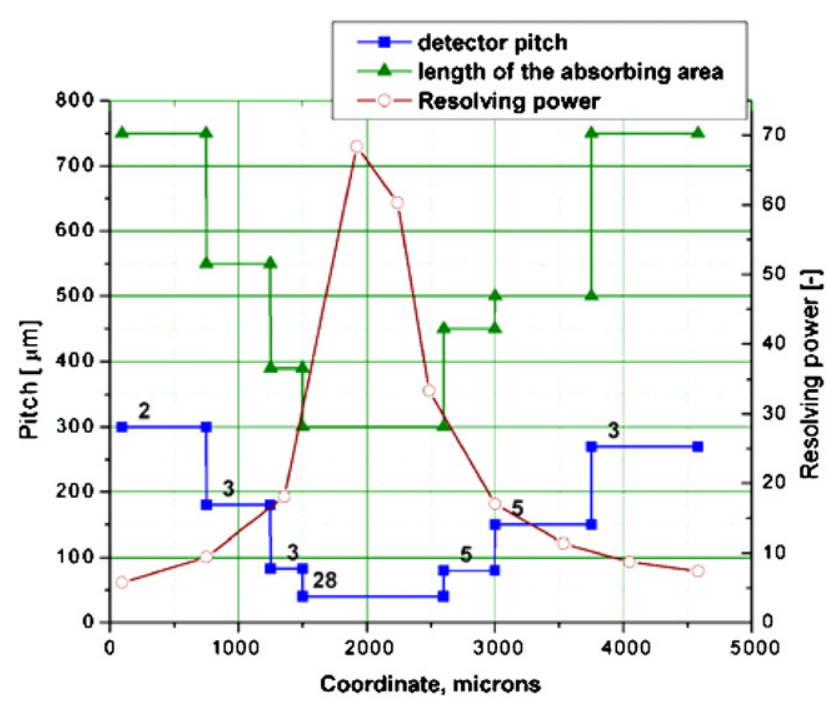

Figure 4. Length of the absorbing area and pitch of the array and spectrometer resolution versus the coordinate along the detector array. Figures on the graph show the number of the elements with equal dimensions.

The relationship between resolving power and geometry of each sensing element including the absorbing area and distance between elements is shown in figure 4 . The detector pitch presents the width of the projected light spot on the detector and the length of the absorbing area presents the length of the projected light spot. This simulation result is achieved by ZEMAX. Furthermore, the thickness of layers is imposed by the fabrication process and is not easily changed. Consequently, the parameters available for optimization are length and width of the thermopile.

Figure 3 shows the configuration of a thermopile on a silicon nitride ( $\mathrm{SiN}$ ) membrane. Thermocouples are based on n-type-p-type polysilicon (PolySi) junctions. The reason to select this combination is the reduced thermal conductivity, which yields a higher temperature difference at any given optical intensity, as compared to the PolySi-metal combination. The sensitivity as the function of the beam length can be obtained by fixing other parameters, such as the width of the thermopile and the spacing between two thermocouples:

$$
S=\frac{N \alpha \Delta T}{E}=\frac{N \alpha}{W \lambda_{\mathrm{eq}} t_{\mathrm{eq}} \times \frac{1}{L}} \quad\left(\mathrm{VW}^{-1}\right),
$$


where the temperature difference between hot and cold junctions is defined as

$$
\begin{aligned}
\Delta T & =T(L)-T_{0}=\frac{E}{G} \\
& =\frac{E}{\left[4 \varepsilon \sigma T_{0}^{3}+\lambda_{\text {gas }}\left(\frac{1}{d_{1}}+\frac{1}{d_{2}}\right)\right] A+W \lambda_{\text {eq }} t_{\mathrm{eq}} \beta \operatorname{coth}(\beta L)} \\
& \times(\mathrm{K}),
\end{aligned}
$$

where $N$ denotes the number of thermocouples, $\alpha$ the Seebeck coefficient, $E$ the radiant flux, $W$ the width of thermocouples, $L$ the length of thermocouples, $t_{\mathrm{eq}}$ the thickness of PolySi, $\lambda_{\text {eq }}$ the equivalent thermal conductance of $\mathrm{SiN}$ and PolySi, $G$ the heat conductance, $\beta$ the heat transfer coefficient, $T_{0}$ the ambient temperature, $\sigma$ the Stefan-Boltzmann constant, $\lambda_{\text {gas }}$ the thermal conductivity of gas, $A$ the absorbing area, $d_{1}$ the distance between the thermocouples and the lower cap and $d_{2}$ the distance between the thermocouples and the upper cap. In equation (1) convection and radiation effects are disregarded.

The response time is an important performance measure for the detector array. It is related to thermal time constant. From the analytical model, we get the following equation for the time constant of the thermopile:

$$
\begin{aligned}
\tau & =\frac{C}{G} \\
& =\frac{V \rho c}{\left[4 \varepsilon \sigma T_{0}^{3}+\lambda_{\text {gas }}\left(\frac{1}{d_{1}}+\frac{1}{d_{2}}\right)\right] A+W \lambda_{\text {eq }} t_{\text {eq }} \beta \operatorname{coth}(\beta L)}
\end{aligned}
$$

where $V$ denotes the volume, $C$ the heat capacitance, $\rho$ the density of the material, $c$ the specific heat of the material, $\beta$ the heat transfer coefficient and $G$ the heat conductance. Increasing the thermal conductance of an element reduces the response time; however, it also reduces the induced temperature difference across the junctions at a given heating power and, hence, degrades sensitivity. Another approach for minimizing time constant is to reduce the thermal capacitance of the structure either by selecting a membrane material with low density and specific heat or by utilizing thinner layers. The time constant increases as the length of the thermopile increases. Therefore, a trade-off between the sensitivity and time constant should be made, which determines the length of a thermopile.

\subsection{Thermopile array design}

The array design is based on an element composed of a bridge structure with the thermopile and is divided into five different groups according to optical requirements of the integrated microspectrometer. For each group a separate optimization and simulation for geometry of elements is performed.

The design starts with the part with the highest optical resolution of 68, group 1, in the middle of the array. Group 1 has 28 elements and the distance between elements is $40 \mu \mathrm{m}$. The length of the absorbing area is defined as $300 \mu \mathrm{m}$ and results from the optical imaging. Since the design is realized using a bridge structure, the length of the absorbing area should be one half, namely $150 \mu \mathrm{m}$, in the cantilever analytical model. The bridge structure is realized by fabrication of a gap

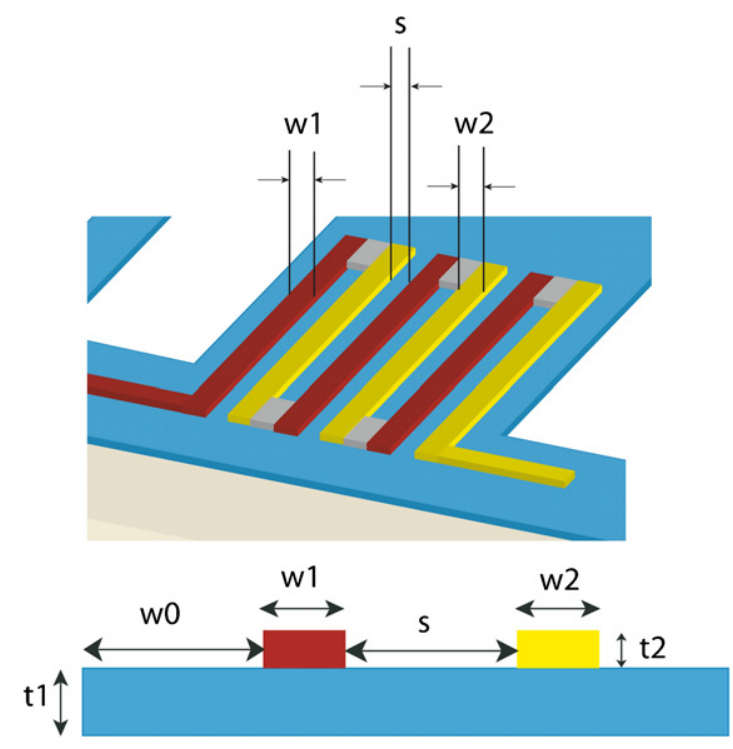

Figure 5. Structure of the thermopile on a SiN membrane.

between elements on the rectangular membrane. The $8 \mu \mathrm{m}$ gap can be achieved by RIE. Since the minimum feature size of implantation is described as $2 \mu \mathrm{m}$, the width of thermal legs is chosen at $4 \mu \mathrm{m}$ for safety. Referring to figure 5 , the space between legs is determined and fixed at $2 \mu \mathrm{m}$. There is also a $2 \mu \mathrm{m}$ wide space between the rim of membrane and the first and the last thermocouple leg. Considering all the required dimensional constraints, the number of thermocouples can only be 2 . The generated voltage is expected to be low since it is proportional to the number of thermocouples. There are two additional layers deposited on the $800 \mathrm{~nm}$ thick SiN membrane. One is the $300 \mathrm{~nm}$ PolySi that constitutes the thermocouple and the other one is the $100 \mathrm{~nm} \mathrm{SiN}$ that insulates the conduction between metal and PolySi. The sensitivity is calculated using the analytical model as $290 \mathrm{~V} \mathrm{~W}^{-1}$.

For other groups, which have lower optical resolution, the design procedure is almost the same. The number of thermocouples can be increased though, which makes it possible to generate bigger voltage and hence increase the sensitivity. Figure 6 shows the relation between the number of thermocouples and sensitivity for group 2 of thermopiles. However, with the increase in the number of thermocouples, the total resistance of the thermopile is also increased which means higher noise and therefore lower detectivity. Specific detectivity is defined as

$$
D^{*}=\frac{S \sqrt{A \Delta f}}{\text { noise }}=\frac{S \sqrt{A \Delta f}}{\sqrt{4 k T_{0} R_{\mathrm{el}}}} \quad\left(\mathrm{cm} \sqrt{\mathrm{Hz}} \mathrm{W}^{-1}\right)
$$

where $S, A, \Delta f, k, T_{0}$ and $R_{\mathrm{el}}$ are the sensitivity defined in equation (1), absorbing area, bandwidth of the amplifier used to measure the detector signal, Boltzmann constant, the ambient temperature and the electrical resistance of thermopiles, respectively. Specific detectivity for thermopiles with a variable number of thermocouples of group 2 type of thermopiles is calculated and the result is shown in figure 6 . The number of thermocouples is chosen to be 4 for group 

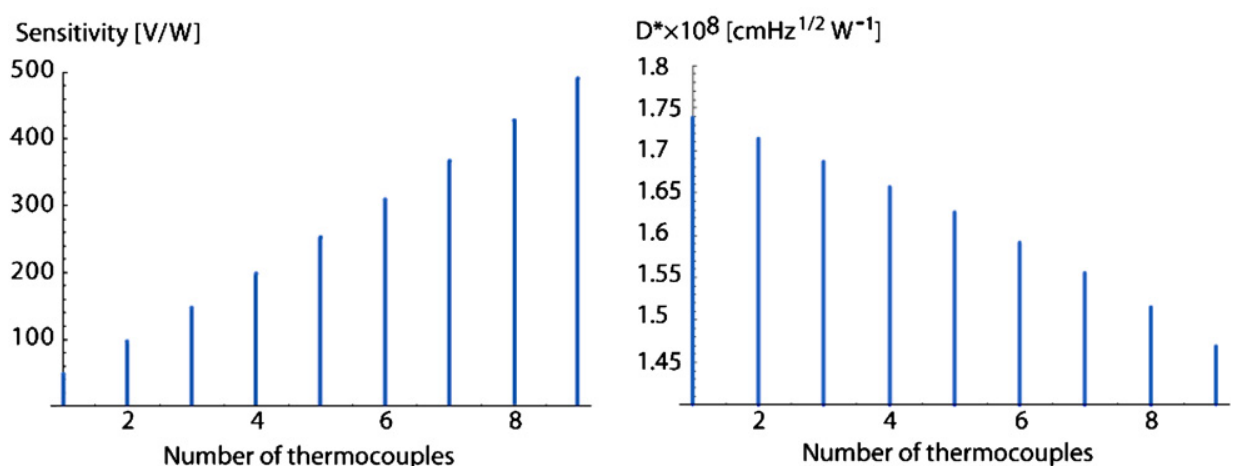

Figure 6. Sensitivity (left) and detectivity (right) as a function of number of thermocouples.

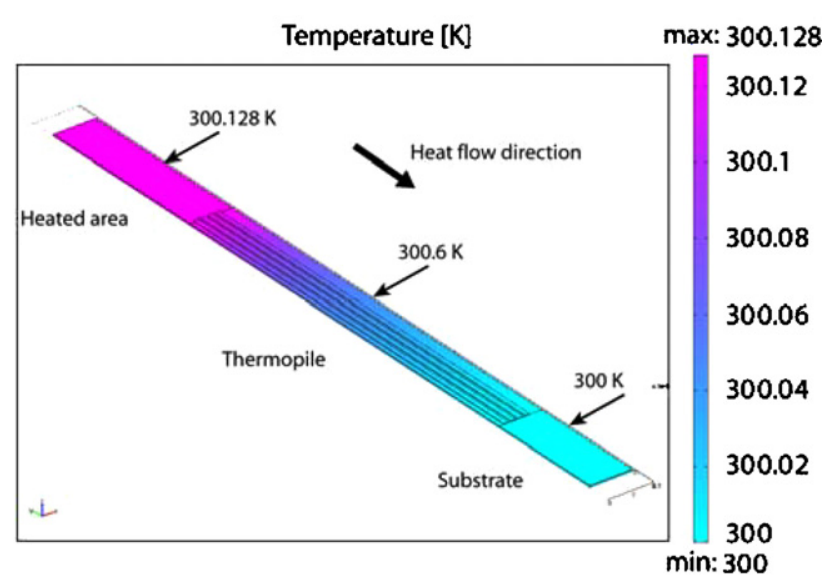

Figure 7. FEM simulation result of a group 1 element with a cantilever (half bridge) structure.

2, which gives a width of $6 \mu \mathrm{m}$ and a specific detectivity of $1.63 \times 10^{8} \mathrm{~cm} \mathrm{~Hz}^{1 / 2} \mathrm{~W}^{-1}$.

\subsection{Numerical verification of the design}

Since the analytical model gives the approximate performance, FEM is used for verification and for finalizing the design parameters. Commercial FEM software, COMSOL, simulates the temperature gradient along the thermopile length from the defined three-dimensional model [13]. Figure 7 shows the 3D FEM simulation result of the temperature distribution along an element in group 1 thermopiles. The model can be reduced to half of the bridge structure due to symmetry. The rectangular shape of the cantilever structure is one half of a bridge. It is $36 \mu \mathrm{m}$ wide and $591 \mu \mathrm{m}$ long $(150 \mu \mathrm{m}$ the length of absorber, $341 \mu \mathrm{m}$ the effective length of the thermopile and $100 \mu \mathrm{m}$ the substrate). The power of $0.27 \mu \mathrm{W}$ is radiated to the surface of the absorber. On the other side of the element, the temperature of the substrate is fixed to $300 \mathrm{~K}$. The figure indicates a temperature difference between hot and cold junctions of the thermopile of about $0.126 \mathrm{~K}$, which is equivalent to a sensitivity of $289 \mathrm{~V} \mathrm{~W}^{-1}$. The simulation result shows a reduced sensitivity as compared to the analytical model, which is due to the fact that the $3 \mathrm{D}$

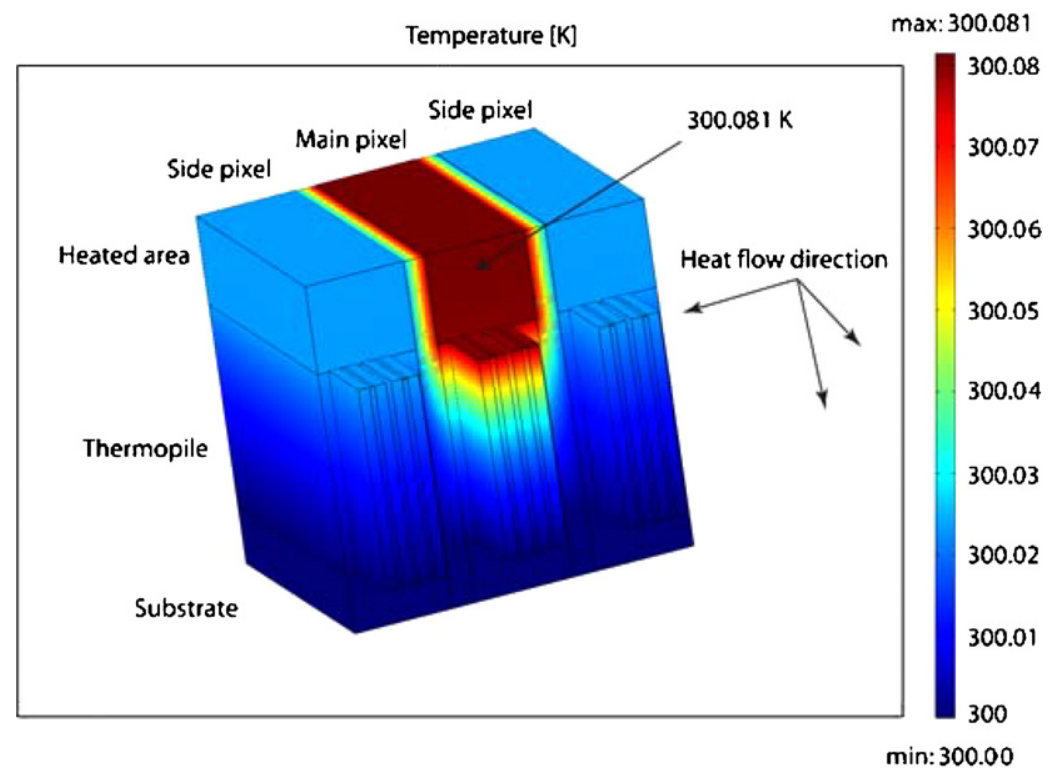

Figure 8. FEM cross-talk simulation on three elements; the gap between elements is $8 \mu \mathrm{m}$. 


\section{Nitride deposition $(0.8 \mu \mathrm{m})$ both sides}

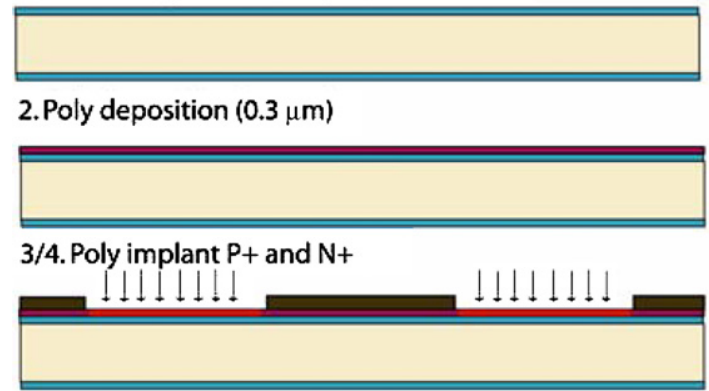

5. Poly Etch

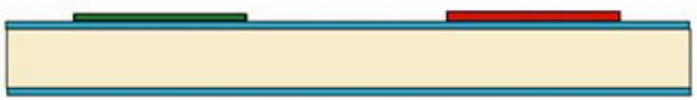

6. Nitride deposition $(0.3 \mu \mathrm{m}) /$ Contact window etch

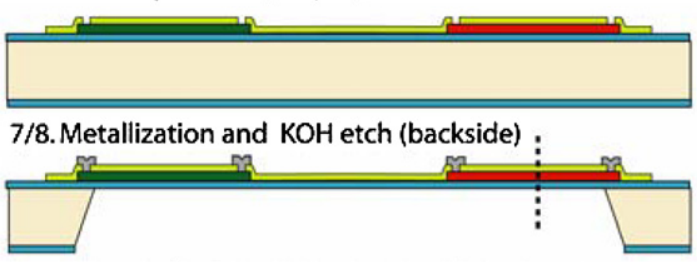

9. RIE etch ( $90^{\circ}$ rotated cross-sectional view)

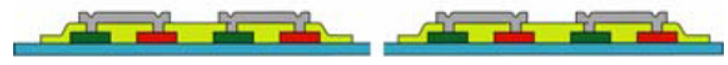

Figure 9. Post-CMOS fabrication sequence (cross-sectional view).

FEM analysis also considers convection and radiation in the complex coordinate system.

The cross-talk between two adjacent elements has also been simulated with COMSOL and the results are shown in figure 8 . The energy leaks to the adjacent element through the gap are by convention in air. It is supposed that the thermal conductivity of air is $0.03 \mathrm{~W} \mathrm{~m}^{-1} \mathrm{~K}^{-1}$. Heat was imposed at one element and the temperature difference is simulated between hot and cold junctions. The temperature difference in the radiated element obtained from the cross-talk simulation is around $0.08 \mathrm{~K}$. Comparison with the simulation result of figure $7(0.126 \mathrm{~K})$ indicates that the ratio is about $63 \%$, which implies that there would be $37 \%$ energy leakage from each

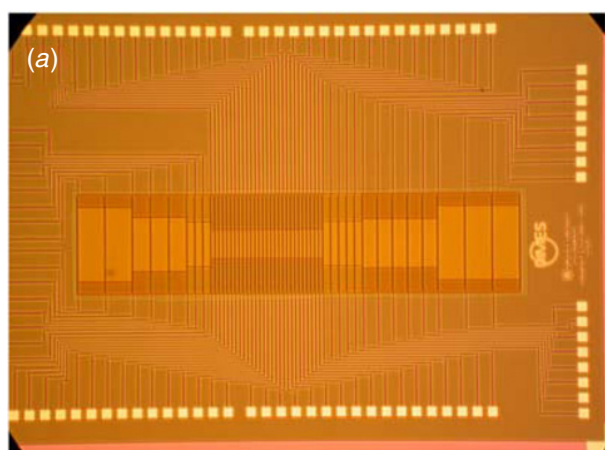

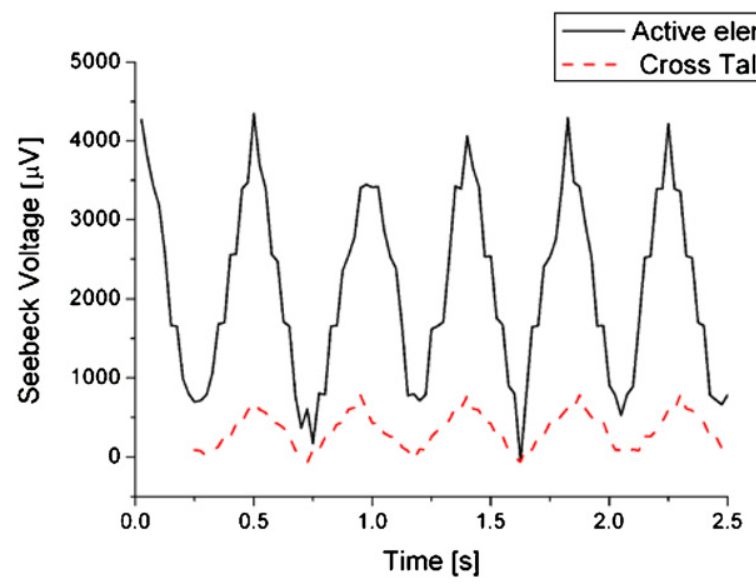

Figure 11. Cross-talk measurement result.

element to neighbouring elements. This means about $19 \%$ cross-talk between each of the two pairs of adjacent elements.

\section{IC-compatible fabrication of the thermopile array}

The fabrication was done in DIMES facility of TUDelft with a standard CMOS process followed by a backside bulkmicromachining step. Six masks are used for the front side processing of the wafer and one additional mask for the micromachining from the backside of the wafer. The process sequence is shown simplified in figure 9.

Wafer processing started with $800 \mathrm{~nm}$ of low stress SiN film formation and then several process steps followed. Firstly, a $300 \mathrm{~nm}$ low-stress PolySi layer was grown by LPCVD. Secondly, boron was implanted at $40 \mathrm{keV}$ and $5 \times 10^{15} \mathrm{~cm}^{-2}$ to realize p-type PolySi. After cleaning procedure, n-type PolySi was formed by phosphorous doping (40 keV and $7.5 \times$ $10^{15} \mathrm{~cm}^{-2}$ ). Thirdly, RIE was applied to remove PolySi from the backside and unwanted PolySi from the front side. In the next step, $300 \mathrm{~nm}$ low-stress SiN was deposited by LPCVD to make the isolation between metal contacts and PolySi. Aluminium was deposited and patterned on top to define connection of thermocouples and bonding pads. Finally,

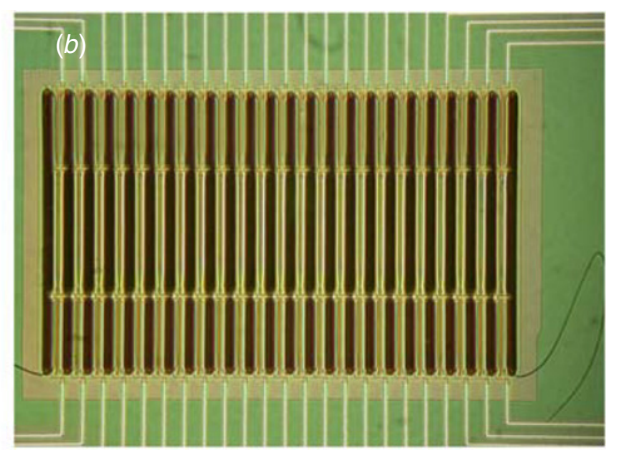

Figure 10. Top view ( $a$ ) of the unequally sized thermopile array and $(b)$ detailed view of one thermopile with an etched membrane for reduced cross-talk. 
the wafer was processed with $\mathrm{KOH}$ wet etching to make the membranes for better thermal isolation. RIE was used to make the gap between thermopiles for limiting cross-talk.

Figure 10 shows the completed thermopile array. The limiting factor is the stress level in the SiN membrane. The mechanical strength of the membrane is significantly reduced after the RIE etch required to thermally isolate the thermocouples, which seriously limits process yield.

\section{Measurement results}

The result of optical measurement of the array based on irradiation of a single element by a pulsed laser with a frequency of $2 \mathrm{~Hz}$ and simultaneous recording of the Seebeck voltages of both that element and the neighbouring ones is shown in figure 11 . The ratio of the voltage with 2 pixels indicates a $20 \%$ cross-talk in the experiment, which is in good agreement with simulation.

One option to decrease cross-talk is to have a wider trench between elements. However, this results in a larger element pitch in the array, which reduces the spectral selectivity. A more effective solution is to operate the array in vacuum, which also contributes to the sensitivity of each thermopile element.

\section{Conclusions}

The design and fabrication of a CMOS compatible thermopile array for use in a miniaturized infrared microspectrometer has been presented. The design of the thermopile detector array is dictated by the optical properties of the imaging grating. The final device structure is based on an analytical and numerical model and is intended for application in the 1.5-3 $\mu \mathrm{m}$ wavelength range. Considering the constraints of a spectral pattern imposed by the microspectrometer diffractive imaging grating, the main dimension of sensing elements could not be fixed. The design parameters optimized are the length and width of thermocouples. After choosing the size and number of thermocouples in each thermopile, the performance can be estimated by the analytical model. Subsequently, simulations have been performed using COMSOL. Resolving power is maximum at the centre element of the array $\left(R=\frac{\lambda_{0}}{\Delta \lambda}=68\right)$ and the calculated sensitivity is $S=289 \mathrm{~V} \mathrm{~W}^{-1}$, when assuming a perfect blackbody absorber. A spacing of $4 \mu \mathrm{m}$ between elements limits the cross-talk between adjacent elements to $19 \%$ theoretically and is verified experimentally to be about $18 \%$. This is not acceptable in a practical device operation. A wider trench between elements helps to decrease cross-talk, but not efficiently. Moreover, the larger element pitch in the array would reduce the spectral selectivity. A more effective solution is to operate the array in a vacuum, which also contributes to the sensitivity of each thermopile element.
The designed array has been fabricated in a standard CMOS process followed by a backside wet etching step to make membranes and a subsequent RIE step to realize beams. The critical factor in the fabrication is stress control in the nitride membrane, which is the main topic of further research.

\section{Acknowledgments}

This work has been supported in part by the Dutch technology foundation STW under grant DET.6667. Devices have been fabricated at the DIMES (Delft Institute of Microsystems and Nanoelectronics). The authors are indebted to $\mathrm{W}$ van der Vlist for his assistance in device fabrication and to Professor P M Shapiro for helpful discussions.

\section{References}

[1] Wolffenbuttel R F 2005 MEMS-based optical mini- and microspectrometers for the visible and infrared spectral range J. Micromech. Microeng. 15 S145-52

[2] Kwa T A and Wolffenbuttel R F 1992 Integrated grating/detector array fabricated in silicon using micromachining techniques Sensors Actuators A 31 259-66

[3] Kong S H and Wolffenbuttel R F 2005 Spectral performance of a micromachined infrared spectrum analyzer in silicon IEEE Trans. Instrum. Meas. 54 264-7

[4] Yen H W, Friedrich H R, Morrison R J and Tangonan G L 1981 Planar Rowland spectrometer for fiber-optic wavelength demultiplexing Opt. Lett. 6 639-41

[5] Goldman D S, White P L and Anheier N C 1990 Miniaturized spectrometer employing planar waveguides and grating couplers for chemical analysis Appl. Opt. 29 4583-9

[6] Sander D and Muller J 2001 Selffocusing phase transmission grating for an integrated optical microspectrometer Sensors Actuators A 88 1-9

[7] Chaganti K, Salakhutdinov I, Avrutsky I and Auner G W 2006 A simple miniature optical spectrometer with a planar waveguide grating coupler in combination with a plano-convex lens Opt. Express 14 4064-72

[8] Avrutsky I, Chaganti K, Salakhutdinov I and Auner G 2006 Concept of a miniature optical spectrometer using integrated optical and micro-optical components Appl. Opt. 45 7811-7

[9] Ura S, Okayama F, Shiroshita K, Nishio K, Sasaki T, Nishihara H, Yotsuya T, Okano M and Satoh K 2003 Planar reflection grating lens for compact spectroscopic imaging system Appl. Opt. 42 175-80

[10] ZEMAX 2000 Optical Design Program, User's Guide, Version 9.0 (Tucson, AZ: Focus Software, Inc.)

[11] Elbel T, Lenggenhager R and Baltes H 1992 Model of thermoelectric radiation sensors made by CMOS and micromachining Sensors Actuators A 35 101-6

[12] Wijngaards D D L and Wolffenbuttel R F 2005 Thermo-electric characterization of APCVD PolySi $\mathrm{Si}_{0.7} / \mathrm{Ge}_{0.3}$ for IC-compatible fabrication of integrated lateral Peltier elements IEEE Trans. Electron Devices 52 1014-25

[13] COMSOL Multiphysics 3.2 2006, COMSOL Inc. http://www.comsol.com 